\title{
On Laminar and Turbulent Boundary Layer in Supersonic Flow
}

\author{
R. LAdenburg and D. Bershader \\ Palmer Physical Laboratory, Princeton University, Princeton, New Jersey
}

$T$ HE variations in refractive index due to compressibility effects in supersonic gas streams permit the use of optical techniques in the experimental study of such flows. The particular suitability of the optical methods in the supersonic regime stems from the fact that, in contrast to mechanical devices such as probes, they do not produce disturbances (shocks) or influence the flow in any way. Qualitative information about density distribution and formation of shocks in the flow can be obtained easily by shadowgrams and schlieren pictures. For quantitative measurements the interferometer is a very suitable instrument, giving directly the density distribution in the flow and, provided the pressure and temperature of the reservoir are known, also the corresponding pressures, temperatures and velocities. Such interferometric studies of supersonic jets have been carried out and published some time ago. ${ }^{1}$

\section{II}

Recently the interferometer has also been applied to a supersonic wind tunnel especially constructed for optical studies. Dry air is obtained from a large pressure tank by means of a sliding valve which opens within about a tenth of a second and discharges the air into an evacuated region consisting of a supersonic nozzle constructed for a Mach number of 2.4, a diffuser and a receiving tank. This arrangement permits a wide variation of pressure and density in the test section. The latter is $3 \times 3$ inches in cross section and contains plane parallel windows through which the light for the optical study of the gas flow can pass. Figure 1 shows the optical path and the Zehnder-Mach interferometer consisting of two plane parallel plates $B$, so-called beam splitters, and two plane mirrors $M$. Also shown is the "compensating chamber" $C C$ for equalizing the optical path length in the two beams. The light source $S$ is a special magnesium spark of less than a microsecond duration (for details see reference 1). In order to obtain pictures at well defined conditions, the spark is released by a pressure switch which is set at a predetermined value so as to make contact shortly after the valve is opened. Preliminary experiments on the distribution of the flow in the test section and of the density distribution within the turbulent boundary layer along the walls of this section have been published elsewhere. ${ }^{2}$

\section{III}

In order to get more reliable information on the physical properties of the boundary layer in supersonic flow, a half-wedge was installed in the test section. This profile, spanning the height of the test section between both windows, was made from quarter inch steel plate, surface ground and tapered on one side to a sharp leading edge of apex angle $\sim 4^{\circ}$. A shadowgram obtained in parallel light (Fig. 2) shows the position of the shocks arising at the leading edge of the half-wedge, also the boundary layers along the surfaces of the plate and on one of the channel walls produced by an air flow of Mach number $M \sim 2.3$. This picture was made at a "stagnation" pressure $P_{0}=249 \mathrm{~cm} \mathrm{Hg}$ (3 atmospheres) in the reservoir, corresponding to a density $^{3}$ of the flowing gas $\rho_{1}=0.62 \mathrm{mg} / \mathrm{cc}$. The bound-

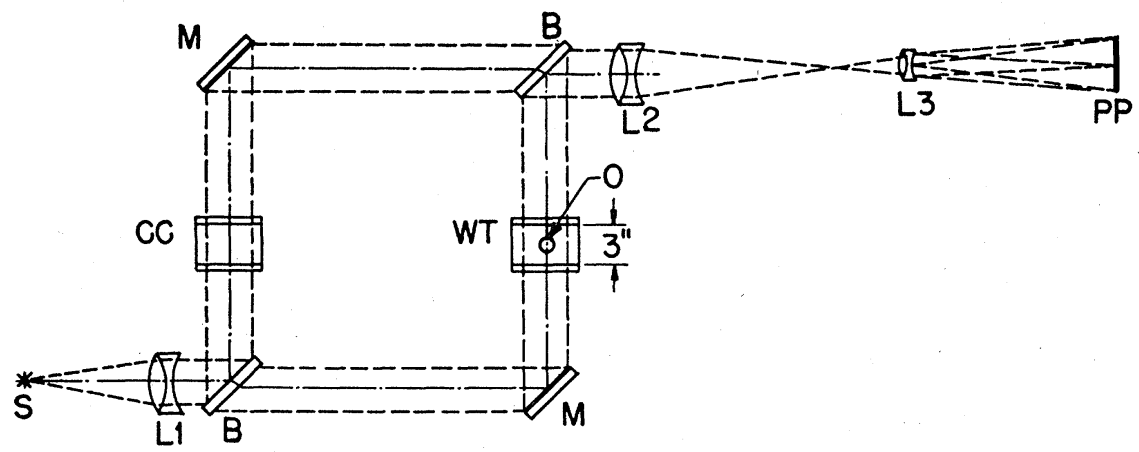

FIG. 1. Optical path for wind tunnel interferometry. $B, B$ are the two beam splitters, $M, M$ the two mirrors of the interferometer, $W T$ is the test section of the wind tunnel containing the object (model) 0 . The light from the spark $S$ made parallel by lens $L 1 ; L 2$ and $L 3$, produce an image of 0 and of the interference fringes upon the photographic plate $P P$.

${ }^{1}$ Ladenburg, Van Voorhis, and Winckler, NAVORD Report 69-46; Phys. Rev. 73, 1359 (1948); J. Winckler, Rev. Sci. Inst. 19, 307 (1948).

${ }^{2}$ R. Ladenburg, Proceedings of the VII International Congress of Applied Mechanics (in press); see also D. Bershader, Rev. Sci. Inst. 20, 260 (1949).

${ }^{3}$ Atmospheric air at N.T.P. has the density $1.29 \mathrm{mg} / \mathrm{cc}$. 
ary layer on the downstream half of the flat surface exhibits a characteristic granular structure (see also Fig. 6), whereas in the upstream half the layer is so thin and has such a high density gradient that a strong refraction occurs.

Figure 3 gives an interference picture under conditions similar to those of Fig. 2, obtained by using monochromatic violet light with the help of suitable light filters. Figure 4 is obtained with unfiltered light from the same spark and shows the corresponding white light fringes which allow one to determine the whole number of fringes shifted in the flow picture with respect to the no-flow picture. The actual fringe shift between flow and no-flow permits one to compute the change of refractive index and therefore the change of density due to the flow (see reference 1). Generally the compensating chamber $C C$ (see Fig. 1) is evacuated to the same density as that expected in the undisturbed flow (the "free stream") of the channel. Therefore, upstream of the leading edge of the plate the fringe shift between the flow and no-flow picture is only a fraction of a full fringe. The shock produced on the flat side of the plate is obviously pretty weak, whereas on the wedge side a much stronger shock occurs. The shock on the flat side is probably due to a slight bluntness of the edge which produces some compression of the flow. However after passing this blunt edge, the flow is expanded again so that the conditions along the flat plate are practically the same as in the undisturbed flow. This is borne out by measurements on the interferometer pictures.

\section{IV}

Our main interest concerns the boundary layer at the flat surface of the plate. The boundary layer is evidenced by a sudden bending of the interference fringes and their crowding within a narrow region, corresponding to an appreciable density decrease and a temperature increase produced by the friction between the high speed gas stream and the gas at rest at the surface of the plate. Figure 5 is an enlarged part of an interference picture, which shows details of the crowded fringes in the boundary layer at the plate. Before the flow the plate itself is at room temperature, equal to the temperature of the reservoir, whereas the undisturbed flow has a much lower temperature corresponding to its adiabatic expansion in the nozzle. By measuring the position of the dark and bright fringes within the narrow boundary layers one obtains the corresponding fringe shifts from which several points of the density profile can then be computed. The higher the pressure the more points are obtained. In this way measurements of the density of the boundary layer along the flat side of the plate could be carried out in a region between $59 \mathrm{~cm} \mathrm{Hg}$ and $250 \mathrm{~cm} \mathrm{Hg}$ pressure in the reservoir corresponding to a test section density variation in the ratio 1 to 4 . The thicknesses of these boundary layers lay in the present case between 0.6 and $1.4 \mathrm{~mm}$.

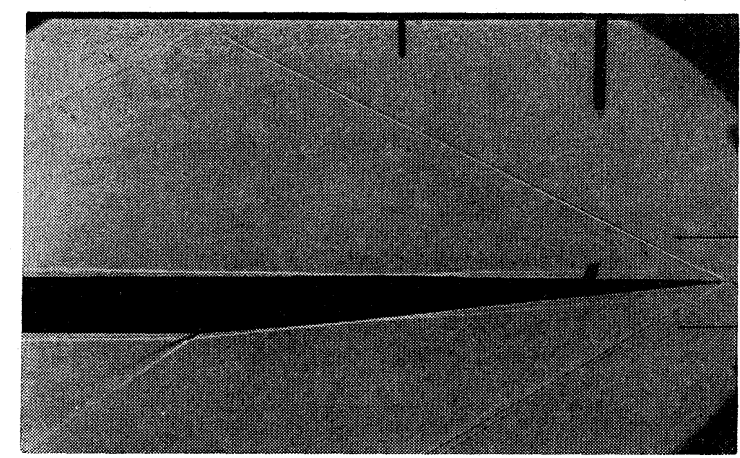

FIG. 2. Shadowgram of half-wedge made in parallel light in a supersonic air stream of $M \sim 2.3$. The rather weak shock at the flat side penetrates into the apparently turbulent boundary layer practically up to the wall of the wind tunnel where it is reflected. The boundary layer along the surfaces of the object is of a turbulent character on the downstream half, but is so thin on the upstream half that strong refraction of the parallel light occurs. (Arrows show the direction of gas flow.)

It was expected that the boundary layer flow would be laminar near the leading edge, growing turbulent further on. This has been borne out qualitatively by the shadowgrams and quantitatively by the evaluation of the interferograms. The apparent transition from laminar to turbulent flow is very evident on shadowgrams such as Fig. 2, obtained by shining parallel light on a photographic plate placed closely adjacent to the test section. Figure 6 is an enlarged part of such a shadowgram, obtained at $P_{0}=119 \mathrm{~cm}$ pressure in the reservoir. One observes a rather sudden change from a smooth line to a diffuse region having a characteristic granular structure, which probably represents turbulent eddies (see arrow marking the apparent transition point in Fig. 6). However, this does not prove that the transition is actually a sharp one (see quantitative results discussed below). Similar transitions have been observed in schlieren pictures of transonic flow by Ackeret ${ }^{4}$ and Liepmann; ${ }^{5}$ the present pictures show perhaps somewhat more detail in the turbulent structure.

\section{V}

Two sets of data have been obtained in the present experiments, representing studies (1) in the upstream part of the flat plate surface at a stagnation pressure $P_{0}=59 \mathrm{~cm}$ and (2) at the downstream end of the plate at a stagnation pressure $P_{0}=249 \mathrm{~cm}$. These two cases appeared to give the closest approximations to laminar and turbulent flow, respectively. Figure 7 gives a typical example of the density distribution obtained from the evaluation of interference fringes in the boundary layer along the flat side of the plate (a) at a distance of about $5 \mathrm{~cm}$ from the leading edge at $P_{0}=59 \mathrm{~cm}$ and (b) at a distance of about $10 \mathrm{~cm}$ from the leading edge

${ }^{4}$ Ackeret, Feldmann, and Rott, Mitteil. Inst. Aerodyn. T. H. Zürich No. 10 (1946); N. A. C. A., Techn. Mem. No. 1113.

5 H. W. Liepmann, J. Aero. Sci. 13, 623 (1946). 


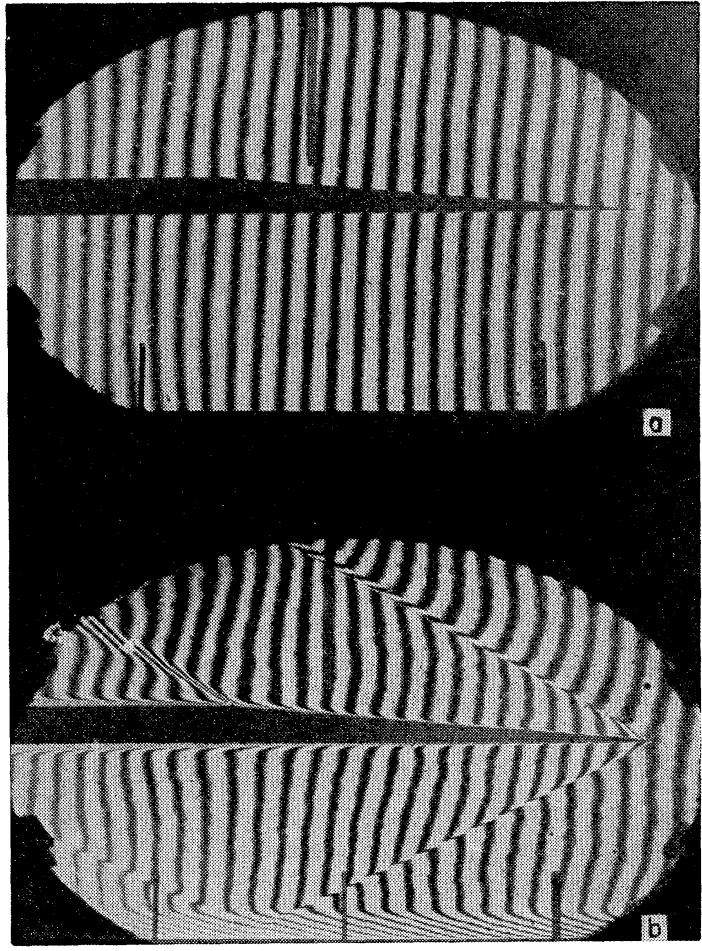

FIG. 3. Interferogram in monochromatic light a) without flow, b) with flow at $M \sim 2.3$; "stagnation" pressure $P_{0}=119 \mathrm{~cm} \mathrm{Hg}$. The shocks show up by discontinuous shifts of the fringes (the double shock at the wedge side of the object is due to the interaction with the boundary layer on the glass walls). The abrupt bending and crowding of the fringes along the wall of the test section (only one of the two walls is visible) is due to the lower density in the boundary layer. A similar effect is observed in the much thinner boundary layers along the surfaces of the object over nearly the whole length. Because of the focusing effect of lenses 2 and 3 (Fig. 1) the large refraction at the upstream part of the object which appeared in the non-focused shadowgram is avoided.

$P_{0}=249 \mathrm{~cm}$. Since the pressure through the boundary layer may be supposed to be constant and equal to the known pressure in the undisturbed flow, the temperature distribution can also be obtained (see Figs. 8 and 9). The temperatures $T$ in units of the free stream temperature $T_{1}$ are given as a function of the distance $y$ from the surface divided by the observed boundary layer thickness $\delta$. This thickness is pretty well defined on shadow and schlieren pictures as well as on interferograms. Figure 8 shows the temperature profile at $P_{0}=59$ $\mathrm{cm}$ for two stations near the leading edge of the plate at relatively low density in the free stream (numerical data are given in the caption to the figure), corresponding to the relatively low Reynolds number $R e_{x}=\left(u_{1} \rho_{1} x\right)$ $\left.\mu_{1}\right)=3.5 \times 10^{5}$ and $4.4 \times 10^{5}$, respectively. Index 1 refers to the free stream; $\mu$ is the viscosity coefficient.

In Fig. 9 the temperature profile is plotted (in the same dimensionless units as in Fig. 8) at $P_{0}=249 \mathrm{~cm}$ for four stations far downstream from the leading edge of the plate at relatively high density in the free stream (for numerical data see the caption to the figure), corresponding to the higher Reynolds numbers $R e_{x}$ between $2.8 \times 10^{6}$ and $3.5 \times 10^{6}$. These Reynolds numbers suggest that the boundary layer flow in Fig. 8 is laminar and in Fig. 9 is turbulent, in agreement with the qualitative conclusions drawn from shadowgrams. In the case of $P_{0}=59 \mathrm{~cm}$ granulation appears only at the downstream end of the surface, about $10 \mathrm{~cm}$ from the leading edge $\left(R e_{x} \sim 9 \times 10^{5}\right)$, whereas at $P_{0}=249 \mathrm{~cm}$ eddies begin to appear at $x=5 \mathrm{~cm}\left(R e_{x} \sim 1.7 \times 10^{6}\right)$ and are rather prominent at the points where the temperatures of Fig. 9 are determined. There is an outspoken difference between the two figures. In Fig. 8 the temperature corresponding to the evaluated points nearest to the wall (about $0.05 \mathrm{~mm}$ away) is only $20^{\circ}$ lower than the stagnation temperature, whereas in Fig. 9 the temperature near the wall is still about $90^{\circ}$ under the stagnation value.

In order to obtain also the velocity profile in the boundary layer some suitable assumptions are necessary. The simplest assumption would be to say that the total energy per unit mass in the boundary layer is constant and equal to that of the undisturbed "free"

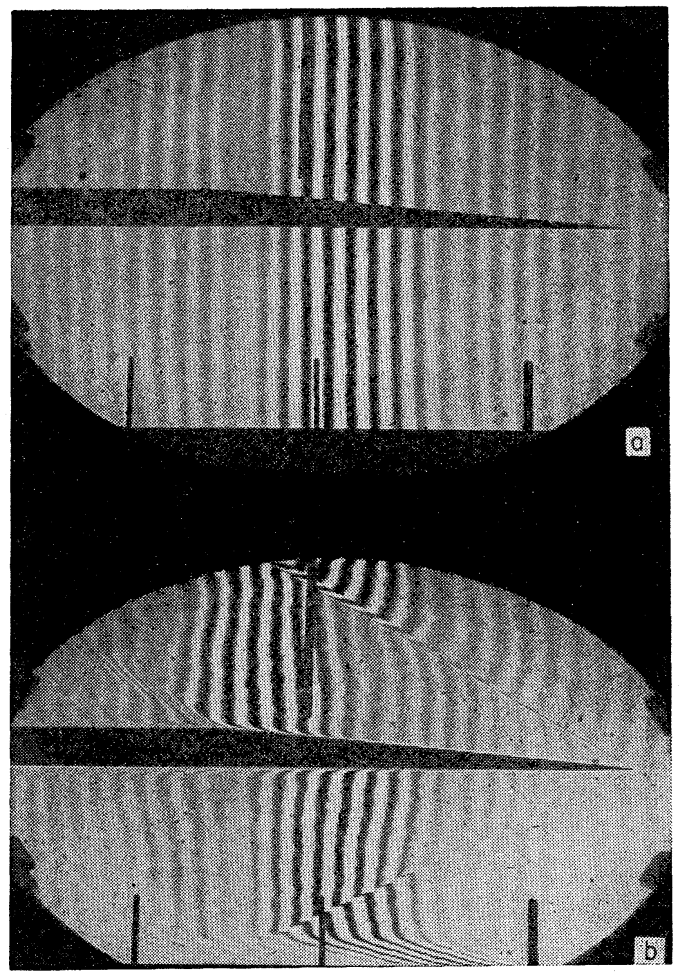

FIG. 4. Interferogram using unfiltered light a) without flow, b) with flow at the same flow conditions as Fig. 3. The white light fringes allow one to number the fringes and so to determine the whole number of fringes shifted. Outside the shocks there is no appreciable fringe shift since the air density in the compensating chamber ( $C C$ of Fig. 1) was set at the expected density of the undisturbed flow. 
gas flow, that is

$$
u^{2} / 2+C_{p} T=u_{1}^{2} / 2+C_{p} T_{1}=C_{p} T_{0},
$$

where $u$ and $T$ are velocity and temperature in the boundary layer, $T_{0}$ is the stagnation temperature and $C_{p}$ the specific heat at constant pressure. Luigi Crocco ${ }^{6}$ has shown that the differential equations for compressible flow, including viscosity and heat conductivity, have Eq. (1) as a particular integral if the Prandtl number

$$
\operatorname{Pr}=C_{p} \mu / k=1 \text { (turbulent flow) }
$$

( $k$ is the coefficient of heat conductivity), provided that no heat is added from external sources; that means that the gas, brought to rest at the surface of an insulated wall, has stagnation temperature. Therefore, as a first approximation we compute the velocity ${ }^{7} u$ in the turbulent boundary layer from the temperature according to Eq. (1), that is, we assume that Eq. (2) holds, and that the temperature of the surface of our metal plate still has the stagnation temperature ${ }^{8} T_{0}$. In this way the velocity profile of Fig. 10 is obtained for the same 4 stations as the temperature profile in Fig. 9, near the downstream end of the flat plate surface for $P_{0}=249 \mathrm{~cm}$. The coordinates in Fig. 10 are in dimensionless units $u / u_{1}$ and $y / \delta$. For the most part the experimental distribution agrees rather well with the simple relation

$$
u / u_{1}=(y / \delta)^{1 / 9},
$$

which is represented by the drawn curve. Only the points nearest to the surface of the plate show an appreciable deviation from this curve. Such an exponential law has been found to hold for turbulent

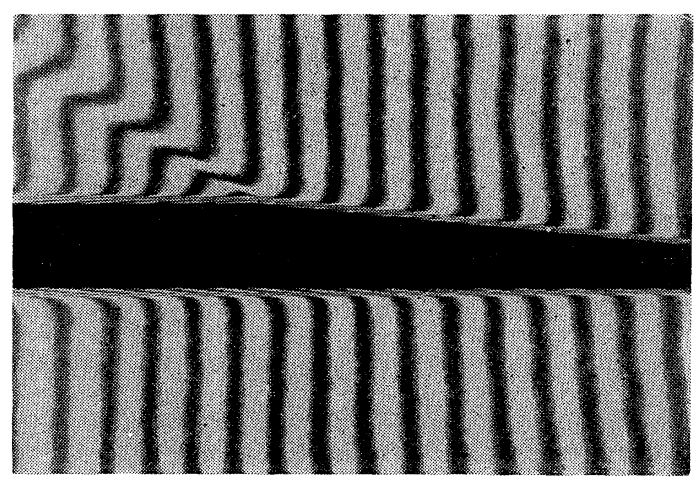

FIG. 5. Enlarged part of an interferogram at $P_{0}=59 \mathrm{~cm} \mathrm{Hg}$ and correspondingly low density in the test section showing the narrow fringes in the boundary layers.

${ }^{6}$ L. Crocco, L'Aerotecnica 12 (No. 2) Febbraio 1932; N.A.C.A. Techn. Mem. No. 690. See also A. Busemann, Handbuch der Experimental Physik (1931), IV, Part 1, 366; and Z.A.M.M. 15 23 (1935).

7 In the velocity so computed the turbulent fluctuations average out.

${ }_{8}$ The flow lasts for only about one second so that there is not much time available for the plate to cool.

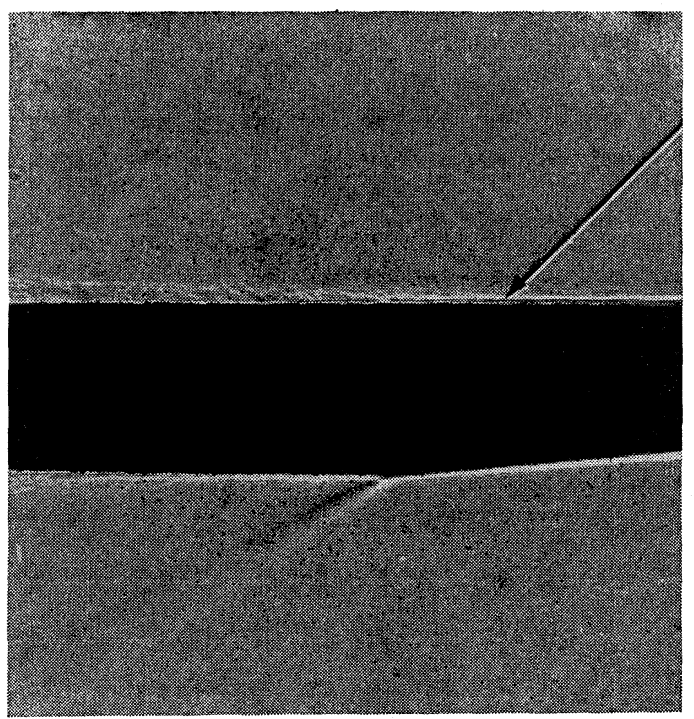

FIG. 6. Enlarged part of a shadowgram in parallel light at $P_{0}=119 \mathrm{~cm} \mathrm{Hg}$. The arrow shows the point in the boundary layer where the turbulent character begins to appear.

boundary layer in an incompressible fluid ${ }^{9}$ where the exponent decreased from $1 / 7$ to $1 / 10$ with increasing Reynolds number. Our former measurements of the velocity distribution in the boundary layer along the wall of the test section (reference 2), also calculated by using Eq. (1), could be represented in a similar way. ${ }^{10}$

Mach number computations show that by far the largest part of the turbulent boundary layers in the present case is supersonic. One finds that even as close as $0.1 \mathrm{~mm}$ to the plate surface $M$ is still about 1.5 . Shadowgrams such as Fig. 2 show that incident oblique shocks penetrate deeply into the boundary layer on the channel wall, curving in accordance with decreasing Mach number. Thus, the drop to zero velocity must occur within a very narrow inner region of the boundary layer which, evidently being of an appreciably different nature from the main supersonic portion, may be categorized as a sub-layer and may well be laminar. In fact, laminar sub-layers have been observed in the study of subsonic turbulent flow through tubes, and found to decrease in thickness with increasing velocity. Therefore, the computation of the velocity for the points nearest to the wall according to Eq. (1) (i.e., assuming $P r=1$ ) may be a poor approximation, since it is known that for non-turbulent flow $P r \simeq 0.72$. Besides, points on the present profiles associated with values of $y / \delta$ less than 0.1 possess a rather large uncertainty in this variable because of a residual uncertainty in the posi-

${ }^{9}$ H. Blasius, Forschungshaft V.D.I. 131, 1 (1913); Th. Von Karmann, Z.A.M.M. 1, 233 (1921).

${ }_{10}$ Recent measurements of boundary layers in the wind tunnel at Dainger Field using hypodermic needles as Pitot tubes have led to power law velocity distributions with the experiment $1 / 7$; see Wilson, Young and Thompson, Defense Research Laboratory, University of Texas, Report D R L-196 (January 1949). 


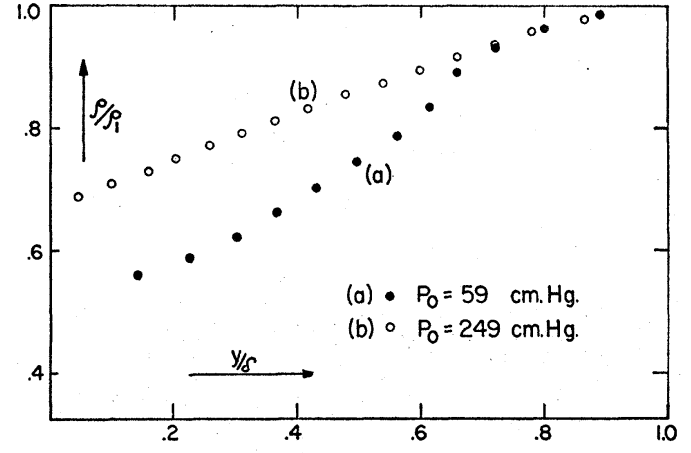

FIG. 7. Point values of boundary layer density as a function of distance $y$ from surface of a flat plate, computed from measured fringe shifts. Ordinates and abscissae are in dimensionless units. (a) Stagnation pressure $P_{0}=59 \mathrm{~cm} \mathrm{Hg}$. Free stream conditions are $\rho_{1}=0.17 \mathrm{mg} / \mathrm{cc}, T_{1}=143^{\circ} \mathrm{K}, M_{1}=2.29, u_{1}=5.4 \times 10^{4} \mathrm{~cm} / \mathrm{sec}$. distance $x$ from leading edge $x=5.1 \mathrm{~cm}, R e_{x}=4.4 \times 10^{5}$. (b) $P_{0}=249$ $\mathrm{cm} \mathrm{Hg.} \rho_{1}=0.62 \mathrm{mg} / \mathrm{cc}, T_{1}=139^{\circ} \mathrm{K}, M_{1}=2.34, u_{1}=5.5 \times 10^{4}$ $\mathrm{cm} / \mathrm{sec} . x=9.5 \mathrm{~cm}, R e_{x}=3.5 \times 10^{6}$.

tion of the plate surface caused by diffraction effects, etc.

On the other hand, since for laminar flow $\operatorname{Pr} \neq 1$, we need another relation to substitute for Eq. (1) in order to compute velocity profiles from the temperature distributions given in Fig. 8. However, for $\operatorname{Pr} \neq 1$, the differential equations for flows with viscosity and heat conductivity do not possess a simple solution. ${ }^{11}$ Study of the equations shows that the physical parameters of the flow, e.g., specific heat, viscosity, etc., appear only in the Prandtl number and in the combination $\rho \mu$, the

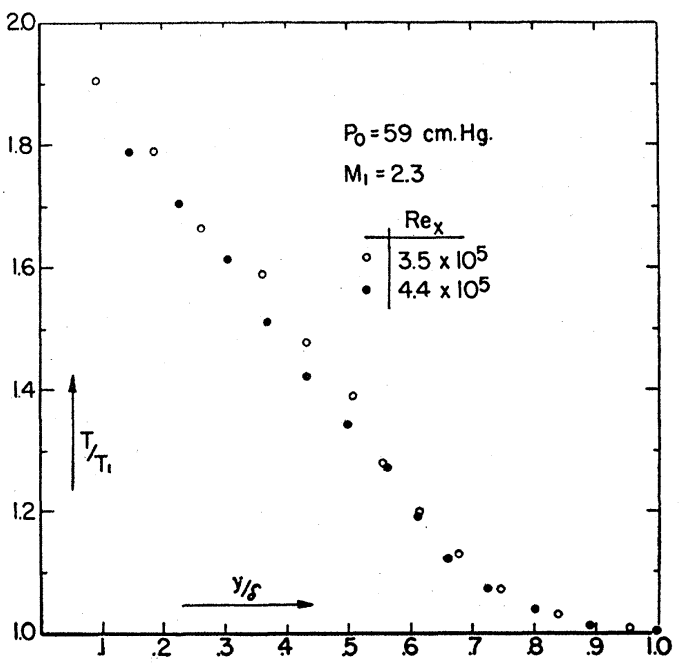

Fig. 8. Composite boundary layer temperature profile for $x=4.1 \mathrm{~cm}$ and $5.1 \mathrm{~cm}$ at conditions corresponding to Fig. 7 (a). Ordinates give temperatures in units of the free stream temperature $T_{1}$. The abscissa is distance from surface $y$, divided by boundary layer width $\delta(\delta=0.60$ and $0.65 \mathrm{~mm}$, respectively). Stagnation temperature $T_{0}=294^{\circ} \mathrm{K}$

" See for example H. W. Emmons and J. G. Brainerd, J. App. Mech. 8, A-105 (1941); W. F. Cope and D. R. Hartree, Phil. Trans. Royal Soc. (A)241, 1 (1948). product of density and viscosity. ${ }^{12}$ The equations are greatly simplified if one assumes a linear dependence between $\mu$ and $T$, very nearly fulfilled in the low temperature regions as investigated here. In this case the product $\rho \mu$ is constant, and Crocco obtains the simple relation

$$
\begin{aligned}
\left(T / T_{1}\right)-1=\left[\left(T_{p} / T_{1}\right)-1\right] & {\left[1-F_{0}\left(u / u_{1}\right)\right] } \\
& +(\gamma-1) M_{1}^{2} F_{1}\left(u / u_{1}\right),
\end{aligned}
$$

where $T_{p}$ is the temperature on the surface of the plate, $\gamma$ is the ratio of the specific heats, $u_{1}, M_{1}$, and $T_{1}$ are velocity, Mach number and temperature of the free stream; $F_{0}$ and $F_{1}$ are functions of the velocity ratio $u / u_{1}$, which are tabulated for

$$
\operatorname{Pr}=0.725
$$

in Table 4 of reference 12. This Eq. (4) is therefore the required relation for computing the velocity profile for $P_{0}=59 \mathrm{cmHg}$. Since we have not yet measured the actual temperature of the surface of our plate during the flow, ${ }^{13}$ we assume in first approximation that $T_{p}=T_{0}$, the stagnation temperature--probably not a bad approximation. ${ }^{8}$ Velocity ratios $u / u_{1}$ obtained from measured points in the boundary layer at two stations are given in Fig. 11 as small open and full circles, corresponding to the points given in Fig. 8. As abscissae the non-dimensional quantity,

$$
\eta=y\left(\operatorname{Re}_{x}\right)^{\frac{1}{2}} / x,
$$

is used in place of $y / \delta$ since this is the variable commonly used in theoretical studies of laminar boundary

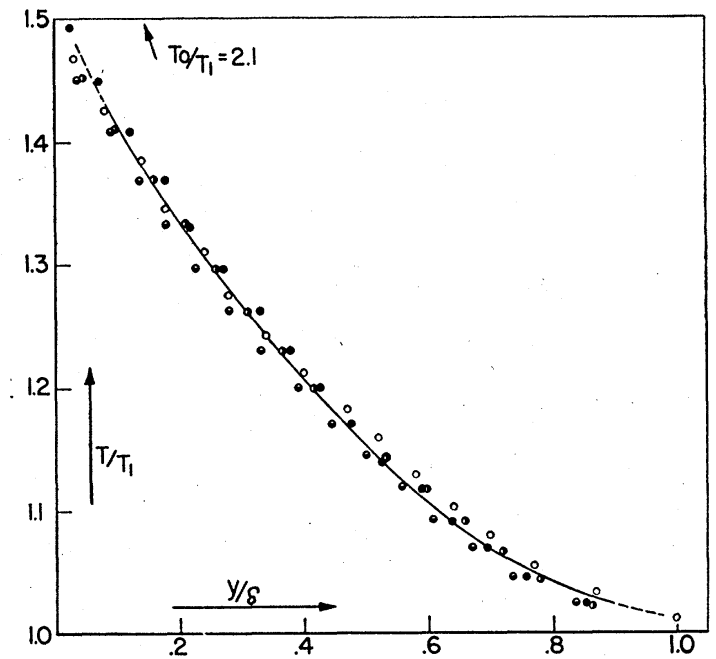

Fig. 9. Composite boundary layer temperature profile for four stations between $x=7.5$ and $9.5 \mathrm{~cm}$, under conditions of Fig. 7(b). Ordinates and abscissae are as in Fig. 8. Values of $\delta$ range between 1.0 and $1.4 \mathrm{~mm} ; T_{0}=293^{\circ} \mathrm{K}$.

${ }^{12}$ L. Crocco, Rendiconti del Circolo Matematico di Palermo, February 9, 1941

${ }_{13}$ Footnote added in proof. Recent preliminary measurements yielded the result that during one second of flow, the temperature of the surface of the plate dropped by about one degree C. 


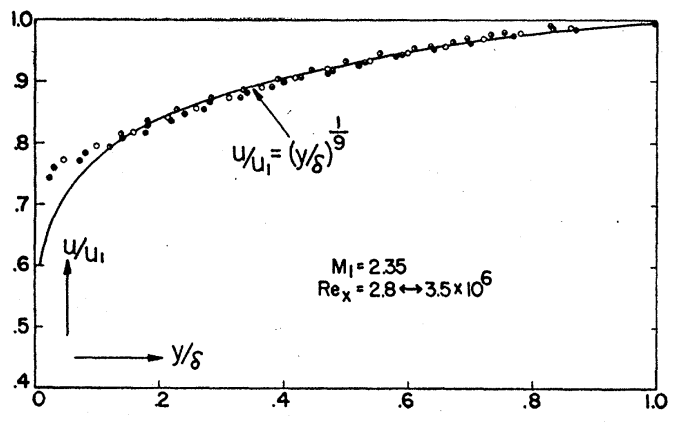

Frg. 10. Composite boundary layer velocity profiles for stations whose temperature curve is given in Fig. 9. Ordinates are velocities $u$ in terms of the free stream velocity $u_{1}$; abscissae are the same as in the Figs. 8 and 9. The drawn curve represents the relation $u / u_{1}=(y / \delta)^{1 / 9}$.

layers. For comparison two curves are shown in Fig. 11: the dashed curve is the well known Blasius distribution $^{14}$ for incompressible laminar flow, as function of $\eta$. The solid curve is the velocity distribution computed from Crocco's theory, ${ }^{12}$ for the case $\rho \mu=$ const. with $M_{1}=2.3$ and $T_{1}=143^{\circ} \mathrm{K}$ and $T_{p}=T_{0}=294^{\circ} \mathrm{K}$. The experimental points lie clearly above the Crocco curve for small values of $\eta$ and reach this curve only for $\eta>5$. The reason for this discrepancy may be either the refraction due to the density gradient in the boundary layer-not yet taken into account in these preliminary measurements - or the fact that the assumptions made in the derivation of the theory are not fulfilled in our experiments.

From the observed velocity profile one obtains for the "displacement thickness" $\delta^{*}=\int_{0}^{\delta}\left(1-\left(u \rho / u_{1} \rho_{1}\right)\right) d y$ the values $0.024 \mathrm{~cm}$ and $0.027 \mathrm{~cm}$ at the two stations $;^{15}$ they are similar to the values of $\delta$ for the stations investigated at $P_{0}=249 \mathrm{~cm}$ (Fig. 10), which lie between 0.024 and 0.031 . The corresponding values of the Reynolds number computed with $\delta^{*}$ as a characteristic length, instead of with $x$, are

for $P_{0}=59 \mathrm{~cm} \mathrm{Hg}$ :

$$
R e_{\left(\delta^{*}\right)}=\sim 2100 \text { (laminar flow) }
$$

for $P_{0}=249 \mathrm{~cm} \mathrm{Hg}$ :

$$
R e_{\left(\delta^{*}\right)}=\sim 9500 \text { (turbulent flow). }
$$

Mach number computations show that in these laminar boundary layers the flow is also predominantly supersonic and becomes subsonic only for $y<0.15 \mathrm{~mm}$, $y / \delta \sim 0.2$.

Nothing definite can be said at present about the transition from laminar to turbulent supersonic flow. In fact, computations of the velocity ratio $u / u_{1}$ as a function of $y / \delta$ for successive stations farther downstream in the case $P_{0}=59 \mathrm{~cm}$, using again Eq. (4),

\footnotetext{
${ }^{14}$ H. Blasius, Zeits. f. Math. u. Phys. 56, 1 (1908); K. Pohlhausen, Zeits. f. Angew. Math. and Mech. 1, 261 (1921).

${ }_{15}$ These values are about twice as large as the values of displacement thickness for incompressible laminar flow $\delta^{*}=1.73 x$ / $\left(R e_{x}\right)^{\frac{1}{2}}$, which would be 0.011 and 0.013 in the two cases.
}

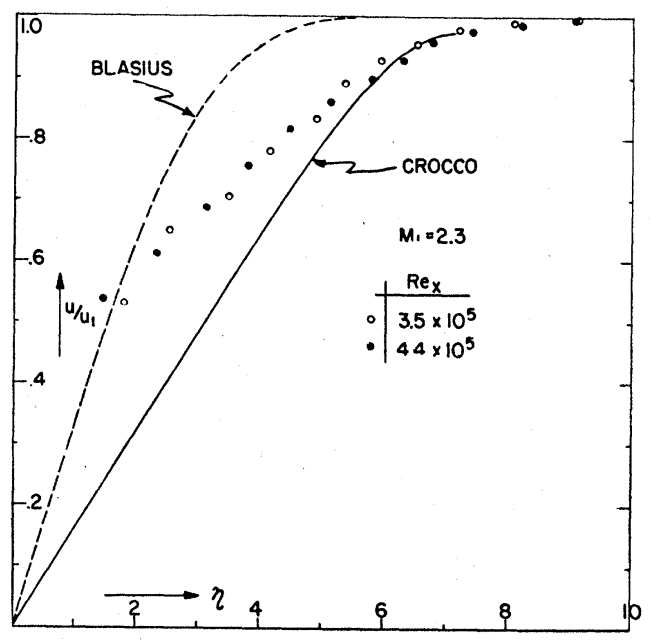

FIG. 11. Composite velocity distribution for the two stations of Fig. 8. Ordinates are velocities $u$ in terms of $u_{1}$, while the abscissa $\eta=y\left(\operatorname{Re}_{x}\right)^{1} / x$. The dashed curve represents the Blasius distribution for laminar incompressible flow. The profile computed from Crocco's theory is given by the solid curve.

show a gradual change in direction of a steeper profile and approach to some extent the power law distribution at $R e_{x}=9 \times 10^{5}$. This gives the impression that associated with the transition from laminar to turbulent flow there is an intermediate region before the turbulent character of the boundary layer manifests itself fully. ${ }^{16}$ Therefore one is not justified in the present case in speaking of a definite transition point, in spite of the impression given by shadowgrams such as Figs. 2 and 6. How this transition region depends upon the turbulent nature of the main flow, on the sharpness and smoothness of the leading edge of the plate, etc.-as the transition region of incompressible flow does-has not yet been investigated.

\section{VI}

The conclusions we may draw from these preliminary investigations are that in supersonic flow at the conditions investigated here, the boundary layer along a plane surface has a turbulent character for $R e_{x}>2 \times 10^{6}$. The velocity profiles obtained follow a power law similar to that observed in incompressible flow. For $R e_{x}<6 \times 10^{5}$ the velocity profile is very different and not far from the theoretical profile computed by Crocco for laminar boundary layers at high velocities.

The present work was performed under a contract with the Office of Naval Research.

The valuable assistance of Dr. C. C. Van Voorhis in performing the experiments and of Miss A. Kenny in evaluating the results are gratefully acknowledged. We are especially indebted to Professor Lester Lees for stimulating discussions on boundary layer theory.

${ }_{16}$ The assumption has been made throughout this work that the external pressure gradient $\Delta p / \Delta x$ is zero. Actually, small local pressure gradients do exist in some regions but they are very small and irregular $\left[(\Delta p / \Delta x)(1 / p)\right.$ is of order $\left.0.01 \mathrm{~cm}^{-1}\right]$. 


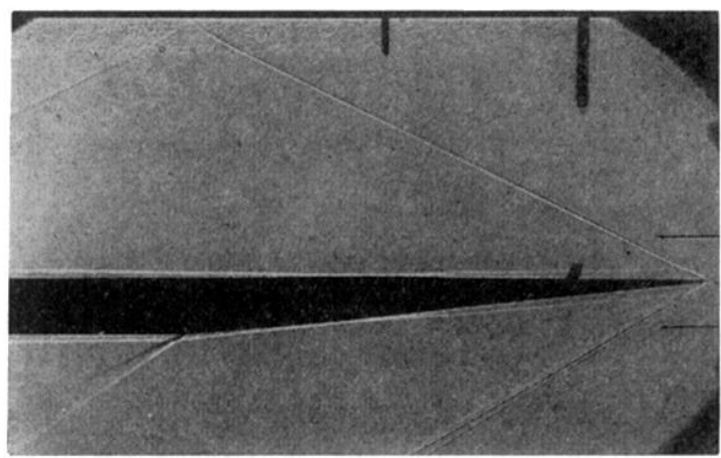

FIG. 2. Shadowgram of half-wedge made in parallel light in a supersonic air stream of $M \sim 2.3$. The rather weak shock at the flat side penetrates into the apparently turbulent boundary layer practically up to the wall of the wind tunnel where it is reflected. The boundary layer along the surfaces of the object is of a turbulent character on the downstream half, but is so thin on the upstream half that strong refraction of the parallel light occurs. (Arrows show the direction of gas flow.) 


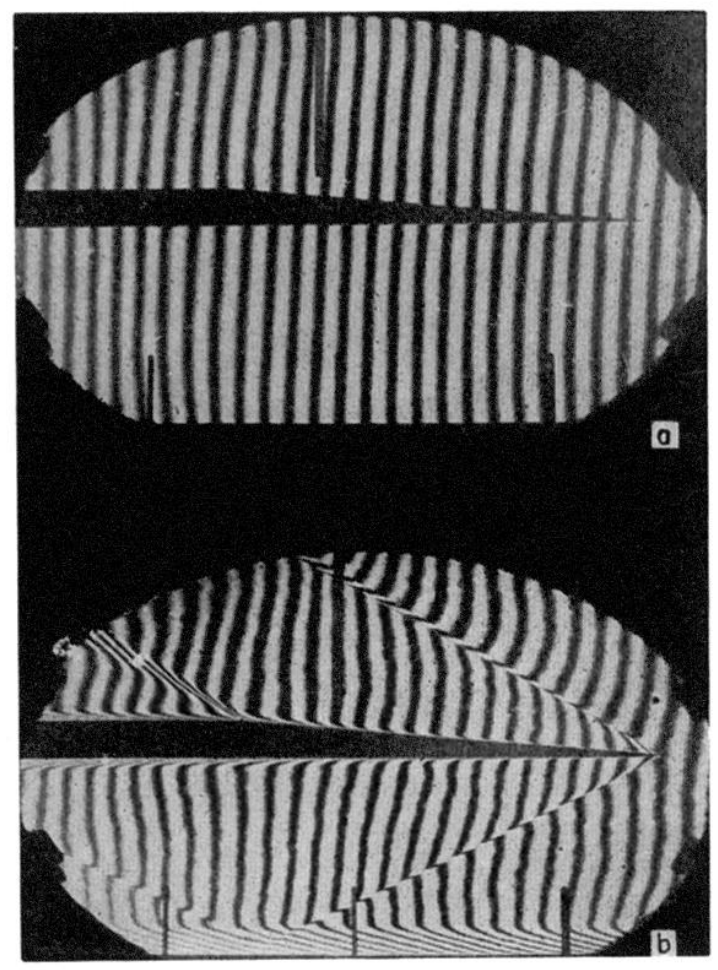

FIG. 3. Interferogram in monochromatic light a) without flow, b) with flow at $M \sim 2.3$; "stagnation" pressure $P_{0}=119 \mathrm{~cm} \mathrm{Hg}$. The shocks show up by discontinuous shifts of the fringes (the double shock at the wedge side of the object is due to the interaction with the boundary layer on the glass walls). The abrupt bending and crowding of the fringes along the wall of the test section (only one of the two walls is visible) is due to the lower density in the boundary layer. A similar effect is observed in the much thinner boundary layers along the surfaces of the object over nearly the whole length. Because of the focusing effect of lenses 2 and 3 (Fig. 1) the large refraction at the upstream part of the object which appeared in the non-focused shadowgram is avoided. 


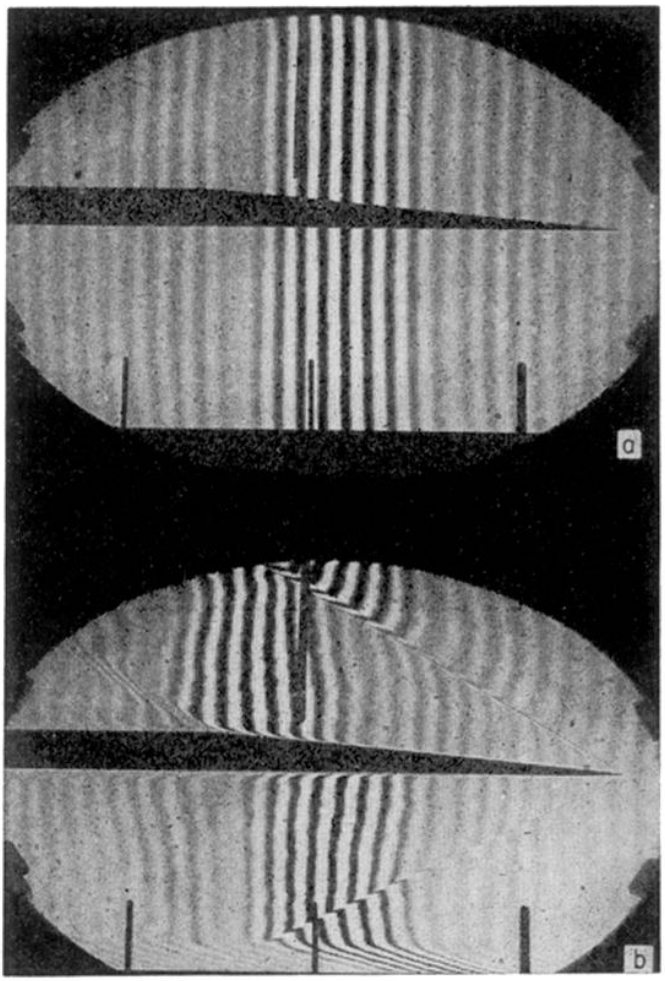

FIG. 4. Interferogram using unfiltered light a) without flow, b) with flow at the same flow conditions as Fig. 3 . The white light fringes allow one to number the fringes and so to determine the whole number of fringes shifted. Outside the shocks there is no appreciable fringe shift since the air density in the compensating chamber ( $C C$ of Fig. 1) was set at the expected density of the undisturbed flow. 


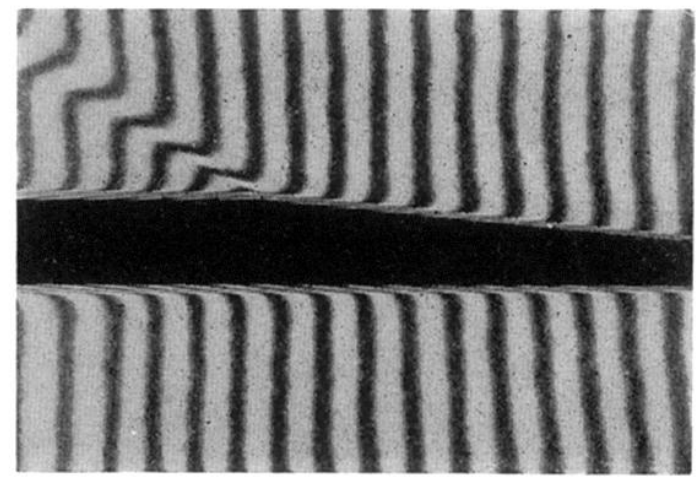

FIG. 5. Enlarged part of an interferogram at $P_{0}=59 \mathrm{~cm} \mathrm{Hg}$ and correspondingly low density in the test section showing the narrow fringes in the boundary layers. 


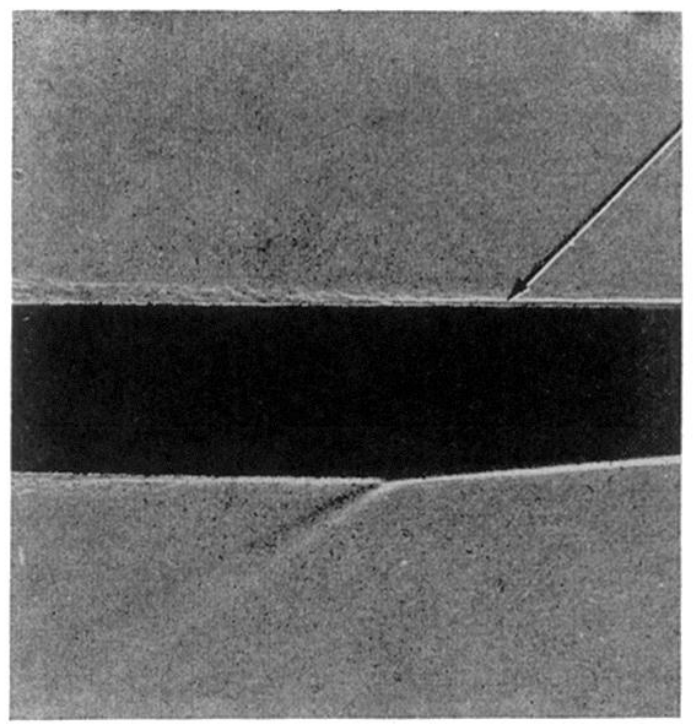

Fig. 6. Enlarged part of a shadowgram in parallel light at $P_{0}=119 \mathrm{~cm} \mathrm{Hg}$. The arrow shows the point in the boundary layer where the turbulent character begins to appear. 